\title{
A rational risk assessment for intravesical recurrence in primary low-grade Ta bladder cancer: A retrospective analysis of 245 cases
}

\author{
MASAKAZU AKITAKE ${ }^{1}$, KEIJIRO KIYOSHIMA ${ }^{1}$, AKIRA YOKOMIZO ${ }^{2}$, \\ KENICHIRO SHIGA ${ }^{2}$, HIROFUMI KOGA ${ }^{2}$, ARIO TAKEUCHI ${ }^{1}$, MASAKI SHIOTA ${ }^{1}$, JUNICHI INOKUCHI ${ }^{1}$, \\ KATSUNORI TATSUGAMI $^{1}$, AKITO YAMAGUCHI ${ }^{2}$ and MASATOSHI ETO ${ }^{2}$ \\ ${ }^{1}$ Department of Urology, Graduate School of Medical Sciences, Kyushu University, Fukuoka 812-8582; \\ ${ }^{2}$ Department of Urology, Harasanshin Hospital, Fukuoka 812-0033, Japan
}

Received January 8, 2018; Accepted March 30, 2018

DOI: $10.3892 /$ mco.2018.1602

\begin{abstract}
The aim of the present study was to evaluate the prognostic impact of size and number of tumors in primary low-grade (LG) Ta bladder urothelial carcinoma (UC), and thus allow accurate risk stratification of low-risk non-muscle invasive bladder cancer (NMIBC). This study was a retrospective analysis of 245 patients with primary LG Ta UC of the urinary bladder who were treated with transurethral resection. Differences in intravesical recurrence-free survival (RFS) according to various cutoff values of tumor size and tumor number were calculated using Cox proportional hazards model. Median maximum size of tumor was $1.4 \mathrm{~cm}$, and 153 patients $(62.4 \%)$ had solitary tumors. Forty-nine patients experienced intravesical recurrence during a median 34 months of follow-up. Patients with solitary tumors had significantly longer RFS times compared with those with $\geq 8$ tumors $(\mathrm{P}=0.003)$. Patients with larger tumors had significantly shorter RFS times for each cutoff value $(\mathrm{P}=0.01$ for $1.0 \mathrm{~cm}, \mathrm{P}<0.0001$ for 1.5 and $2.0 \mathrm{~cm}, \mathrm{P}=0.006$ for $3.0 \mathrm{~cm}$ ). On multivariate analysis, each cutoff value of tumor size was found to be a predictor of RFS; among them, the cutoff of $1.5 \mathrm{~cm}$ showed the strongest association (hazard ratio, 4.12; 95\% confidence interval, 2.11-8.81; $\mathrm{P}<0.001)$. If we consider only lower risk NMIBC patients, such as primary LG Ta, the appropriate cutoff value of tumor size to predict intravesical recurrence might be $1.5 \mathrm{~cm}$, but not $3.0 \mathrm{~cm}$ generally adopted in various guidelines. These findings suggest the need for rational risk assessment with consideration of the diversity of patients with NMIBC.
\end{abstract}

Correspondence to: Dr Keijiro Kiyoshima, Department of Urology, Graduate School of Medical Sciences, Kyushu University, 3-1-1 Maidashi, Higashi-ku, Fukuoka 812-8582, Japan

E-mail: kkiyo@uro.med.kyushu-u.ac.jp

Key words: bladder cancer, low-grade urothelial carcinoma, risk classification, tumor size

\section{Introduction}

Non-muscle invasive bladder cancer (NMIBC) is subdivided into recurrence/progression risk groups according to various clinical and pathological characteristics. Urologists choose adjuvant or therapeutic intravesical instillation after transurethral resection of bladder tumor (TURBT) or radical cystectomy by reference to these risk classifications. Generally, the criteria for lowest risk tumors are restricted to primary, solitary, small, and histologically low-grade (LG) Ta tumors. Low-risk NMIBC is defined as primary, solitary, Ta, LG/G1, size $<3 \mathrm{~cm}$, no carcinoma in situ (CIS) by European Association of Urology (EAU) (1); as LG, solitary, $\mathrm{Ta}, \leq 3 \mathrm{~cm}$ by American Urological Association (AUA)/Society of Urologic Oncology (SUO) (2); and as solitary, primary LG Ta by International Bladder Cancer Group (IBCG) (3). Single instillation of chemotherapeutic agent is recommended as postoperative adjuvant therapy in these low-risk NMIBC patients; however, the definitions of low-risk NMIBC are not consistent among the guidelines.

Most primary and solitary LG Ta tumors are relatively small. So, it is unclear whether the generally adopted cutoff size of $3 \mathrm{~cm}$ is really appropriate in these tumors, because this cutoff value was derived from randomized controlled trials (RCTs) involving NMIBC patients who had diverse clinical and pathological characteristics including biologically more aggressive tumors such as recurrent and/or high-grade tumors. Similarly, the cutoff value of tumor number to appropriately predict the risk is not clear in these populations.

In the current study, we analyzed patients with only primary LG Ta tumors and examined the cutoff values of tumor size and tumor number to appropriately select low-risk patients.

\section{Patients and methods}

Patients. We reviewed the clinical and pathological records of consecutive patients who underwent TURBT for primary bladder cancer from January 2010 to June 2015, and who were histologically diagnosed as LG Ta UC at Kyushu University Hospital and Harasanshin Hospital. Patients with prior and/or concurrent history of upper urinary tract UC and those lacking records of clinical data were excluded. A total of 245 patients 
were included in the final analysis. Histological diagnoses were based on both the WHO classification 2004 (4) and WHO classification 1973 (5). This was an institutional review boards-approved study, and recruitment and protection of patient data were performed according to the approved protocols.

Follow-up evaluations consisted of cystoscopy and urine cytology performed 3 months after TURBT. If no recurrence was seen, the same evaluations were performed every 3 months for 2-3 years, and every 6 months thereafter.

The relationships between clinicopathological characteristics, especially cutoff value of tumor size and tumor number, and clinical outcome in terms of recurrence-free survival (RFS) were examined. Tumor recurrence was defined as identification of a new tumor in the bladder that was confirmed by histological examination of consequent TURBT. Concerning progression-free survival (PFS), only one patient experienced tumor progression, defined as intravesical recurrence with confirmed histological proper muscle invasion or detectable distant metastasis, thus we did not analyze the relationship between tumor progression and clinicopathological features.

Statistical analysis. Statistical analyses were performed with JMP Pro version 12 (SAS Institute, Tokyo, Japan). Actuarial RFS and PFS were calculated by Kaplan-Meier analysis, and univariate comparisons between groups were assessed by log-rank tests. Univariate and multivariate analysis were performed using a Cox proportional hazards model to identify the variables that predict prognostic outcomes. Values of $\mathrm{P}<0.05$ were considered to be statistically significant.

\section{Results}

Patient characteristics. Patient characteristics are shown in Table I. All bladder tumors were histologically diagnosed as LG UC according to the 2004 WHO classification (4), and 91 (37.1\%) were G1 and 154 (62.9\%) were G2 according to the 1973 WHO classification (5). Tumor number was distributed as follows: single tumor in 153 patients (62.5\%); 2-7 tumors in 78 patients (31.8\%); and 8 or more tumors in 14 patients (5.7\%). Median size of maximum tumor was $1.4 \mathrm{~cm}$ in diameter (range, $0.2-6.0 \mathrm{~cm}$ ), and 45 patients $(18.4 \%$ ) had tumors $\geq 3.0 \mathrm{~cm}$ in diameter. A total of 107 patients (43.7\%) received induction intravesical chemotherapy postoperatively. Chemotherapeutic agents used were either epirubicin (Epi-ADM) or a combination of mitomycin C (MMC) and cytarabine (Ara-C), as chosen by the urologist in charge. No patients received Bacille de Calmette et Guérin (BCG) instillation therapy.

Recurrence-free survival analysis. Forty-nine patients (24.1\%) experienced intravesical recurrence in the follow-up period. The RFS of all patients is shown in Fig. 1. Kaplan-Meyer analysis revealed RFS of $88.1 \%$ at 1 year, $80.3 \%$ at 2 years, and $76.7 \%$ at 3 years. On univariate analyses, tumor number $\geq 8(\mathrm{P}=0.03)$, tumor size $\geq 1.0 \mathrm{~cm}(\mathrm{P}=0.01)$, tumor size $\geq 1.5 \mathrm{~cm}$ $(\mathrm{P}<0.0001)$, tumor size $\geq 2.0 \mathrm{~cm}(\mathrm{P}<0.0001)$, and tumor size $\geq 3.0 \mathrm{~cm}(\mathrm{P}=0.006)$ were significantly associated with shorter RFS (Table II). On multivariate models, RFS was shorter in patients with tumor size $\geq 1.5 \mathrm{~cm}$ [hazard ratio (HR) $4.12,95 \%$
Table I. Patient and tumor characteristics.

\begin{tabular}{lcc}
\hline & No. of cases & $\%$ \\
\hline Cases & 245 & - \\
Median age (year, range) & $69(37-90)$ & - \\
Sex & & \\
Male & 200 & 81.6 \\
Female & 45 & 18.4 \\
No. of tumors & & \\
1 & 153 & 62.5 \\
$2-7$ & 78 & 31.8 \\
$>8$ & 14 & 5.7 \\
Grade (WHO 1973) & & \\
G1 & 91 & 37.1 \\
G2 & 154 & 62.9 \\
Median tumor size (cm, range) & $1.4(0.2-6.0)$ & \\
Tumor size & & \\
$\geq 1.0$ & 188 & 76.7 \\
$\geq 1.5$ & 121 & 49.4 \\
$\geq 2.0$ & 99 & 40.4 \\
$\geq 3.0$ & 45 & 18.4 \\
Introduction intravesical chemotherapy & & \\
Done & 107 & 43.7 \\
$\quad$ Not done & 138 & 56.3 \\
Median follow-up (month, range) & $34(3-73)$ & - \\
\hline
\end{tabular}

confidence interval (CI) 2.11-8.81, $\mathrm{P}<0.001$; Table II]. When the cutoff of tumor size was changed from 1.5 to $1.0 \mathrm{~cm}, 2.0$ or $3.0 \mathrm{~cm}$, all of the cutoff sizes were found to predictors of shorter RFS (tumor size $\geq 1.0 \mathrm{~cm}$ : HR $2.77,95 \%$ CI 1.20-8.03, $\mathrm{P}=0.014$; tumor size $\geq 2.0 \mathrm{~cm}$ : HR $4.01,95 \%$ CI $2.18-7.79$, $\mathrm{P}<0.0001$; tumor size $\geq 3.0 \mathrm{~cm}$ : HR 2.16 , 95\% CI 1.13-3.97, $\mathrm{P}=0.02$; data not shown). However, the HR was highest for tumor size $\geq 1.5 \mathrm{~cm}$. Patients with tumor number $\geq 8$ also tended to have shorter RFS, but this was not statistically significant (HR 2.67, 95\% CI 0.94-6.58, $\mathrm{P}=0.06$; Table II).

Among the above clinicopathological variables, we selected two variables for risk stratification in patients with primary LG Ta UC: tumor number $\geq 8$ and tumor size $\geq 1.5 \mathrm{~cm}$ based on the results of multivariate analyses. The patients were classified into three groups as follows: Group 1, patients with a single tumor and maximum tumor diameter less than $1.5 \mathrm{~cm}$; group 3, patients with 8 or more tumors and maximum tumor diameter $1.5 \mathrm{~cm}$ or larger; group 2, patients who did not belong to group 1 or group 3 . These three groups showed significantly different RFS (Fig. 2) $(\mathrm{P}<0.0001)$.

\section{Discussion}

Most of the guidelines for NMIBC are based on evidence from many kinds of clinical trials. For example, EAU guidelines for NMIBC are derived from evidence concerning cutoff values of tumor size and tumor number from seven RCTs that compared prophylactic treatments after TURBT 
Table II. Univariate and multivariate analyses for intravesical recurrence.

\begin{tabular}{|c|c|c|c|c|c|c|}
\hline & \multicolumn{3}{|c|}{ Univariate } & \multicolumn{3}{|c|}{ Multivariate } \\
\hline & HR & $95 \% \mathrm{CI}$ & P-value & HR & $95 \% \mathrm{CI}$ & P-value \\
\hline \multicolumn{7}{|l|}{ Age } \\
\hline$\leq 69$ (reference) & 1 & & & 1 & & \\
\hline$\geq 70$ & 0.62 & $0.31-1.14$ & 0.12 & 0.67 & $0.34-1.23$ & 0.2 \\
\hline \multicolumn{7}{|l|}{ Sex } \\
\hline Male (reference) & 1 & & & - & - & - \\
\hline Female & 0.86 & $0.37-1.74$ & 0.7 & - & - & - \\
\hline \multicolumn{7}{|l|}{ Grade } \\
\hline G1 (reference) & 1 & & & 1 & & \\
\hline $\mathrm{G} 2$ & 1.02 & $0.58-1.85$ & 0.93 & 1.06 & $0.59-1.95$ & 0.85 \\
\hline \multicolumn{7}{|l|}{ Tumor numbler } \\
\hline Single (reference) & 1 & & & - & - & - \\
\hline Multiple & 1.61 & $0.91-2.82$ & 0.1 & - & - & - \\
\hline \multicolumn{7}{|l|}{ Tumor numbler } \\
\hline 1 (reference) & 1 & & & 1 & & \\
\hline $2-7$ & 1.38 & $0.73-2.52$ & 0.31 & 1.36 & $0.69-2.62$ & 0.37 \\
\hline $8-$ & 3.05 & $1.14-6.94$ & 0.03 & 2.67 & $0.94-6.58$ & 0.06 \\
\hline \multicolumn{7}{|l|}{ Tumor size $(\mathrm{cm})$} \\
\hline$\leq 0.9$ (reference) & 1 & & & - & - & - \\
\hline$\geq 1.0$ & 2.82 & $1.23-8.15$ & 0.01 & - & - & - \\
\hline \multicolumn{7}{|l|}{ Tumor size $(\mathrm{cm})$} \\
\hline$\leq 1.4$ (reference) & 1 & & & 1 & & \\
\hline$\geq 1.5$ & 4.28 & $2.22-9.07$ & $<0.0001$ & 4.12 & $2.11-8.81$ & $<0.001$ \\
\hline \multicolumn{7}{|l|}{ Tumor size (cm) } \\
\hline$\leq 1.9$ (reference) & 1 & & & - & - & - \\
\hline$\geq 2.0$ & 4.04 & $2.22-7.77$ & $<0.0001$ & - & - & - \\
\hline \multicolumn{7}{|l|}{ Tumor size (cm) } \\
\hline$\leq 2.9$ (reference) & 1 & & & - & - & - \\
\hline$\geq 3.0$ & 2.43 & $1.30-4.35$ & 0.006 & - & - & - \\
\hline \multicolumn{7}{|c|}{ Induction intravesical chemotherapy } \\
\hline Not done (reference) & 1 & & & 1 & & \\
\hline Done & 1.48 & $0.85-2.63$ & 0.17 & 0.9 & $0.47-1.74$ & 0.75 \\
\hline
\end{tabular}

$\mathrm{HR}$, hazard ratio; CI, confidence interval.

in stage Ta, T1, and Tis bladder cancer patients carried out by the European Organization for Research and Treatment of Cancer (EORTC) (1,6-12). The seven RCTs consisted of 2,596 NMIBC patients who had diverse clinicopathological characteristics composed of not only solitary small-sized low-grade Ta tumors but also multiple large-sized high-grade T1 tumors. In AUA/SUO guidelines, risk categories are not based on a meta-analysis or original studies but represent the panel's consensus regarding the likelihood of recurrence and progression (2); however, the background seems to be based on literature for NMIBC patients with various risks for recurrence and progression.

We formed a hypothesis that data collected from only NMIBC patients with lower risk for recurrence and progression would classify the risk differently from analyses of all NMIBC patients. In the current study, all cutoff points of tumor size: $1.0,1.5,2.0$ and $3.0 \mathrm{~cm}$, were significant predictors for shorter RFS, however, the cutoff point of $1.5 \mathrm{~cm}$ showed the highest risk (HR 4.12, 95\% CI 2.11-8.81, $\mathrm{P}<0.001$ ). In addition, as the median tumor size of the current study was $1.4 \mathrm{~cm}$ it is meaningful to use a cutoff point for tumor size of $1.5 \mathrm{~cm}$ in NMIBC patients with lower risk.

Golabesk et al analyzed 704 cases of primary bladder UC with G1-2 Ta/T1 disease. In this case series, 414 patients (58.9\%) had tumors $>1.5 \mathrm{~cm}$ and 290 (41.1\%) had tumors $\leq 1.5 \mathrm{~cm}$; those with tumor $>1.5 \mathrm{~cm}$ had a significantly higher recurrence rate $(66.7 \%$ vs. $53.6 \%, \mathrm{P}=0.001)$ during a median follow-up period of 64.9 months (13). These results suggest 


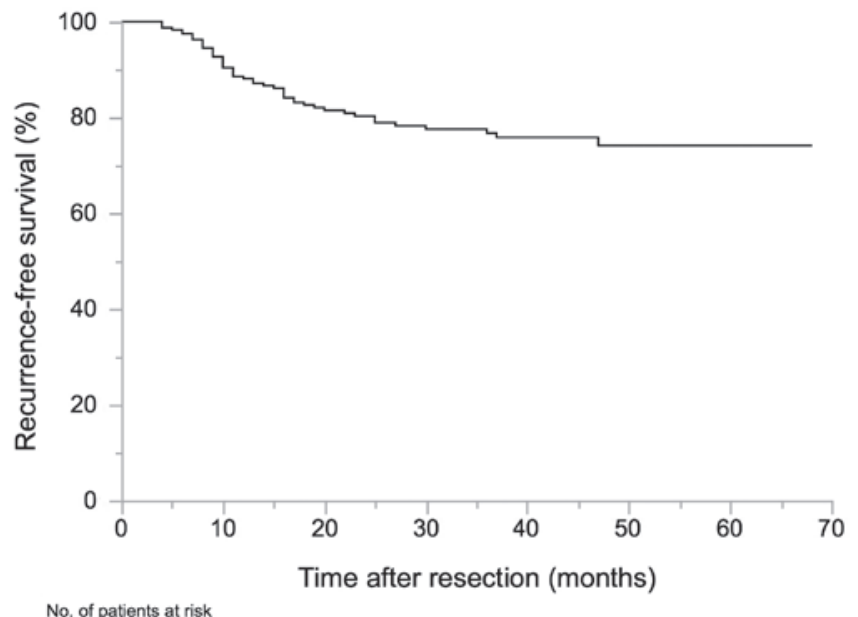

\begin{tabular}{lrrrrrr} 
No. of patients at risk & & & & & & \\
\hline Time (months) & 0 & 12 & 24 & 36 & 48 & 60 \\
all patients & 245 & 188 & 126 & 93 & 40 & 11 \\
\hline
\end{tabular}

Figure 1. Kaplan-Meyer analysis of all patients revealed recurrence-free survival of $88.1 \%$ at 1 year, $80.3 \%$ at 2 years, and $76.7 \%$ at 3 years.

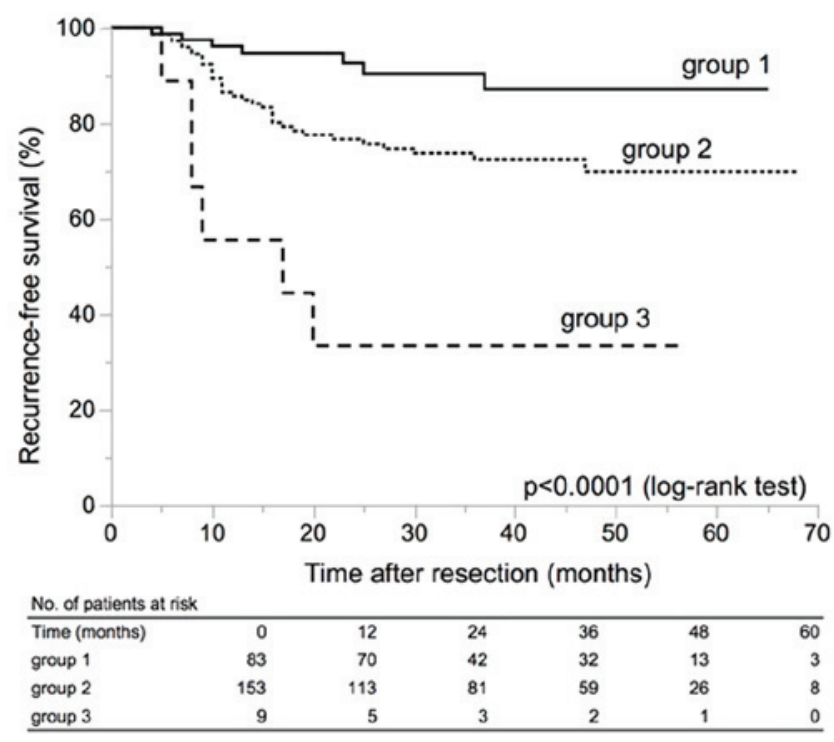

Figure 2. Kaplan-Meyer analysis for recurrence-free survival of subgrouping. The solid line (group 1) shows patients with solitary tumors and tumors smaller than $1.5 \mathrm{~cm}$. The dashed line (group 3) shows patients with 8 or more tumors and tumors $1.5 \mathrm{~cm}$ or larger. The dotted line (group 2) shows patients with data not covered in the solid or dashed lines. These three groups showed significantly different RFS $(\mathrm{P}<0.0001)$.

that tumor size of $1.5 \mathrm{~cm}$ could be an appropriate cutoff in patients with primary LG Ta bladder UC.

Regarding the tumor number, we did not find a significant difference in intravesical RFS between patients with single tumor and those with multiple tumors; however, in a comparison among patients with single tumor, 2-7 tumors, and 8 or more tumors in a similar manner to the EORTC risk table (6), those with 8 or more tumors seemed to have a tendency for shorter intravesical RFS than those with single tumor. Thus, we inferred that tumor multiplicity is likely to have an impact to intravesical recurrence, even in the restricted to patients with primary LG Ta tumors.

In the current study, we did not find a significant difference in intravesical RFS according to histological grade (WHO
1973 G1 vs. G2). There is no discussion about the difference between G1 and G2 in the EORTC report (6). In the newest WHO classification (WHO 2016), the authors emphasized the substantial advantage of eliminating the ambiguity of the grading system in WHO 1973 (14). Therefore, we consider that there is no need to re-classify LG tumors into G1 or G2 according to the WHO 1973 system.

The National Comprehensive Cancer Network (NCCN) guideline of bladder cancer classifies risk category by only histopathological factors, such as LG Ta, HG Ta, LG T1, HG T1 and CIS, and does not consider clinical factors such as past bladder cancer history, tumor size, or tumor number (15). In a recent report, Klaassen et al proposed that LG Ta bladder cancer should not be classified into an intermediate risk group because of its very low risk of progression, and proposed that the criterion of low-risk NMIBC should be 'all LG Ta (regardless of size, multifocal, recurrence)' (16). As mentioned above, there are some classifications that do not include recurrence, tumor number, and tumor size in the risk criteria. However, it is clear that there is a statistically significant difference in RFS when primary LG Ta cancer is classified by tumor size and number, as shown in Fig. 2 . Similarly, IBCG classified patients with multiple and/or recurrent LG Ta tumors (intermediate risk group) into groups with different recommendations for intravesical adjuvant therapy using several factors composed of number (greater than 1) and size (greater than $3 \mathrm{~cm}$ ) of tumors and timing (recurrence within 1 year) and frequency (more than 1 per year) of recurrence (17). Thus, size and numbers of tumors are such major risk factors that it is important to develop a strategy according to these factors.

In the current study, we did not analyze tumor progression because only one patient showed progression to muscle-invasive disease within a median follow-up period of 34 months. Mariappan and Smith reported that there were no cases that progressed to muscle-invasive disease among 115 cases with primary G1 Ta bladder cancer in a mean follow-up of 19.4 years, although 14 cases (12\%) progressed to G2 or Tis/T1 tumors (18). Similarly, Rieken reported that among 1,436 patients with G1 Ta tumors (601 low-risk patients and 835 intermediate-risk patients), 613 patients $(42.7 \%)$ experienced at least one disease recurrence within a median follow-up of 33.5 months, and 68 (4.7\%) showed progression to muscle-invasive disease within a median follow-up of 67.2 months (19). In the recent study of Golabesk et al, among 704 patients with primary G1-2 Ta/G1-2 T1 tumors, 284 patients (40.3\%) had recurrence but only $8(1.1 \%)$ progressed to muscle-invasive disease within a median follow-up of 64.9 months (13). Thus, patients with primary LG Ta bladder cancer rarely show progression to muscle-invasive disease even during a long follow-up period. Consequently, we should understand the characteristics of primary LG Ta bladder cancer, i.e., not always low risk for recurrence but always low risk for progression.

There are several limitations in the current study. First, the analysis was performed retrospectively and the cohort size is not sufficiently large. Second, we did not perform central pathology analyses. Third, there were no definite criteria for performing induction intravesical chemotherapy. Indication of additional induction therapy was individually decided 
by each urologist in charge according to patients and tumor characteristics.

These limitations might lead to some selection bias, however, we showed the prognostic significance of tumor size, in particular a cutoff size of $1.5 \mathrm{~cm}$. Among patients with primary LG Ta bladder cancer, patients with single tumor and tumor smaller than $1.5 \mathrm{~cm}$ have a far lower risk for recurrence, thus postoperative single instillation of chemotherapeutic agents is enough to prevent recurrence. On the other hand, patients with tumors $\geq 1.5 \mathrm{~cm}$ have such a significantly high recurrence risk; thus, another prophylactic treatment should be considered to decrease the recurrence risk.

\section{Conclusion}

We described the criteria for selection of the lowest risk patients among those with low-grade (LG) Ta bladder urothelial carcinoma (UC). If we consider only the lower risk NMIBC patients, the appropriate cutoff value of tumor size to predict intravesical recurrence might be $1.5 \mathrm{~cm}$, which is smaller than $3.0 \mathrm{~cm}$ generally adopted in major NMIBC guidelines. On the other hand, the tumor number was not independent recurrence predictor, however, patients with tumor number $\geq 8$ tended to have shorter RFS in these lower risk NMIBC patients. Our findings suggest the need for rational risk assessment with consideration of the diversity of NMIBC.

\section{Acknowledgements}

The authors would like to thank Dr Mary Derry for editing a draft of this manuscript.

\section{Funding}

No funding was received.

\section{Availability of data and materials}

All data generated or analyzed during this study are included in this published article.

\section{Author's contributions}

MA, KK and AYo designed the study, and MA wrote the initial draft of the manuscript. KS, HK, AT and MS contributed to data collection and interpretation. JI, KT, AYa and ME contributed to analysis and interpretation of data. AYa and ME critically reviewed the manuscript. All authors read and approved the final manuscripts.

\section{Ethics approval}

This study was institutional review boards-approved, and recruitment and protection of patient data were performed according to the approved protocols.

\section{Consent for publication}

Not applicable.

\section{Competing interests}

The authors declare that they have no competing interests.

\section{References}

1. Babjuk M,Böhle A, Burger M,Capoun O,Cohen D, CompératEM, Hernández V, Kaasinen E, Palou J, Rouprêt M, et al: EAU guidelines on non-muscle-invasive urothelial carcinoma of the bladder: Update 2016. Eur Urol 71: 447-461, 2017.

2. Chang SS, Boorjian SA, Chou R, Clark PE, Daneshmand S, Konety BR, Pruthi R, Quale DZ, Ritch CR, Seigne JD, et al: Diagnosis and treatment of non-muscle invasive bladder cancer: AUA/SUO Gudeline. J Urol 196: 1021-1029, 2016.

3. Brausi M, Witjes JA, Lamm D, Persad R, Palou J, Colombel M, Buckley R, Soloway M, Akaza $\mathrm{H}$ and Böhle A: A review of current guidelines and best practice recommendations for the management of nonmuscle invasive bladder cancer by the International Bladder Cancer Group. J Urol 186: 2158-2167, 2011.

4. Eble JN, Sauter G, Epstein JI and Sesterhenn IA: World health organization classification of tumours. In: Pathology and Genetics of Tumours of the Urinary System and Male Genital Organs. IARC Press, Lyon, 2004.

5. Mostofi FK, Sobin LH and Torloni H: Histological typing of urinary bladder tumours. In: International Classification of Tumours 10. World Health Organization, Geneva, 1973.

6. Sylvester RJ, van der Meijden AP, Oosterlinck W, Witjes JA, Bouffioux C, Denis L, Newling DW and Kurth K: Predicting recurrence and progression in individual patients with stage Ta T1 bladder cancer using EORTC risk tables: A combined analysis of 2596 patients from seven EORTC trials. Eur Urol 49: 466-477, 2006.

7. Newling DW, Robinson MR, Smith PH, Byar D, Lockwood R, Stevens I, De Pauw M and Sylvester R: Tryptophan metabolites, pyridoxine (vitamin B6) and their influence on the recurrence rate of superficial bladder cancer. Eur Urol 27: 110-116, 1995.

8. Bouffioux CH, Denis L, Oosterlinck W, Viggiano G, Vergison B, Keuppens F, De Pauw M, Sylvester R and Cheuvart B: Adjuvant chemotherapy of recurrent superficial transitional cell carcinoma: Results of a European Organization for Research on Treatment of Cancer randomized trial comparing intravesical instillation of thiotepa, doxorubicin and cisplatin. J Urol 148: 297-301, 1992.

9. Kurth K, Tunn U, Ay R, Schröder FH, Pavone-Macaluso M, Debruyne F, ten Kate F, de Pauw M and Sylvester R: Adjuvant chemotherapy for superficial transitional cell bladder carcinoma: Long-term results of a European Organization for Research and Treatment of Cancer randomized trial comparing doxorubicin, ethoglucid and transurethral resection alone. J Urol 158: 378-384, 1997.

10. Bouffioux $\mathrm{CH}$, Kurth $\mathrm{KH}$, Bono A, Oosterlinck W, Kruger CB, De Pauw M and Sylvester R: Intravesical adjuvant chemotherapy for superficial transitional cell bladder carcinoma: Results of 2 European Organization for Research and Treatment of Cancer randomized trials with mitomycin $\mathrm{C}$ and doxorubicin comparing early versus delayed instillations and short-term versus long-term treatment. J Urol 153: 934-941, 1995.

11. Witjes JA, v d Meijden AP, Collette L, Sylvester R, Debruyne FM, van Aubel A and Witjes WP: Long-term follow-up of an EORTC randomized prospective trial comparing intravesical bacille Calmette-Guerin-RIVM and mitomycin C in superficial bladder cancer. Urol 52: 403-410, 1998.

12. Oosterlinck W, Kurth KH, Schröder F, Bultinck J, Hammond B and Sylvester R: A prospective European Organization for Research and Treatment of Cancer Genitourinary Group randomized trial comparing transurethral resection followed by a single intravesical instillation of epirubicin or water in single stage Ta, T1 papillary carcinoma of the bladder. J Urol 149: 749-752, 1993.

13. Golabesk T, Palou J, Rodriguez O, Parada R, Skrobot S, Peña JA and Villavicencio H: Long-term bladder and upper urinary tract follow-up recurrence and progression rates of G1-2 non-muscle-invasive urothelial carcinoma of the bladder. Urology 100: 145-159, 2017. 
14. Moch H, Humphrey PA, Ulbright TM and Reuter VE: WHO Classification of Tumours of the Urinary System and Male Genital Organs. International Agency for Research on Cancer, Lyon, 2016.

15. National Comprehensive Cancer Network: Clinical Practice Guidelines in Oncology: Bladder Cancer. Version 5.2017: National Comprehensive Cancer Network 2017. Available at www.ncen.org/professionals/physician_gls/f_guidelines. asp\#bladder. Accessed November 8, 2017.

16. Klaassen Z and Soloway MS: European association of urology and American urological association/Society of urologic oncology guidelines on risk categories for non-muscle-invasive bladder cancer may lead to overtreatment for low-grade Ta bladder tumors. Urology 105: 14-17, 2017.
17. Kamat AM, Witjes JA, Brausi M, Soloway M, Lamm D, Persad R, Buckley R, Böhle A, Colombel $M$ and Palou J: Defining and treating the spectrum of intermediate risk nonmuscle invasive bladder cancer. J Urol 192: 305-315, 2014.

18. Mariappan P and Smith G: A Surveillance schedule for G1Ta bladder cancer allowing efficient use of check cystoscopy and safe discharge at 5 years based on 125 -year prospective database. J Urol 173: 1108-1111, 2005.

19. Rieken M, Xylinas E, Kluth L, Crivelli JJ, Chrystal J, Faison T, Lotan Y, Karakiewicz PI, Holmäng S, Babjuk M, et al: Long-term cancer-specific outcomes of TaG1 urothelial carcinoma of the bladder. Eur Urol 65: 201-249, 2014. 